\title{
Reflets
}

Revue ontaroise d'intervention sociale et communautaire

\section{Le Réseau des services de santé en français de l'Est (RSSFE) de l'Ontario : relever les défis qui découlent de la restructuration du système de santé}

\author{
Jocelyne Lalonde
}

Volume 5, numéro 2, automne 1999

La santé des francophones de l’Ontario

URI : https://id.erudit.org/iderudit/026274ar

DOI : https://doi.org/10.7202/026274ar

Aller au sommaire du numéro

Éditeur(s)

Reflets : Revue ontaroise d'intervention sociale et communautaire

ISSN

1203-4576 (imprimé)

1712-8498 (numérique)

Découvrir la revue

Citer cet article

Lalonde, J. (1999). Le Réseau des services de santé en français de l’Est (RSSFE) de l'Ontario : relever les défis qui découlent de la restructuration du système de santé. Reflets, 5(2), 156-161. https://doi.org/10.7202/026274ar

Tous droits réservés (C) Reflets : Revue ontaroise d'intervention sociale et communautaire, 1999
Ce document est protégé par la loi sur le droit d'auteur. L'utilisation des services d'Érudit (y compris la reproduction) est assujettie à sa politique d'utilisation que vous pouvez consulter en ligne.

https://apropos.erudit.org/fr/usagers/politique-dutilisation/ 


\section{Le Réseau des services de santé en français de l'Est (RSSFE) de l'Ontario : relever les défis découlant de la restructuration du système de santé}

Jocelyne $L$ alonde

diredrice générale, R SSF E

\section{Les origines du RSSFE}

La prestation de services de santé de qualité en français représente un défi de taille pour les établissements et les organismes de santé de l'Est de l'O ntario. Pensons à la pénurie de professionnels capables de desservir le public dans la langue de M olière et aux inquiétudes soulevées par la restructuration des services de santé dans cette région depuis 1997.

$C$ 'est dans ce contexte que le $R$ éseau de services de santé en français de l'Est de l'O ntario a vu le jour, le 13 août 1997. La Commission de restructuration des services de santé (CR SS) de I'O ntario avait alors enjoint I'H ôpital M ontfort d'assumer le rôle principal dans la mise sur pied de ce nouvel organisme « grâce auquel les francophones de la région d' $\mathrm{O}$ ttawa- $\mathrm{C}$ arleton, et audelà, auront accès à une gamme complète de services de qualité qui leur seront offerts en français » ( $R$ apport sur la restructuration 
des services de santé d'O ttawa- $\mathrm{C}$ arleton, $\mathrm{C}$ ommission de restructuration des services de santé, août 1997).

Ce R éseau comprenait les hôpitaux et d'autres organismes tenus d'offrir des services en français (notamment l'H ôpital d'O ttawa, I'H ôpital pour enfants de l'Est de l'O ntario, les Services de santé des Sœurs de la Charité, le Centre de réadaptation, I'Institut de cardiologie de I'U niversité d'O ttawa) de même que certainesinstitutions d'enseignement post-secondaire (U niversité d'O ttawa et La C ité collégiale). Le R éseau avait pour mandat d'identifier leslacunes dansl'offre des services de santé en français et d'aider les hôpitaux et les organismesà obtenir leur désignation sous la $L$ oi sur les services en français.

Le 13 mai 1998, la Commission décidait que l'aire de rayonnement du $\mathrm{R}$ éseau allait s'étendre au-delà de la région immédiate d'O ttawa- $C$ arleton et inclurait dorénavant les comtés de Stormont, Dundas, G lengarry, Prescott et R ussell. Compte tenu de cette directive, les membres du $R$ éseau ont convenu que leur mission serait d'assurer aux 220000 francophones de l'Est ontarien l'accès à toute la gamme des services offerts dans un système de santé restructuré et intégré.

Le R éseau n'est pas un prestataire de services de santé, ni un groupe de pression, ni une agence de revendication et encore moins, une «police de la langue ». II ne se substitue pas àl'autorité, au rôle ou aux responsabilités des nombreux intervenants du système de santé. II n'est pas un agent régulateur. Sa mission en est une de leadership et d'appui en matière de services de santé en français. II vise à rassembler les établissements et les organismes pour qui l'offre de services de santé en français est une préoccupation quotidienne.

C ette approche a permisau $R$ éseau de compter, dès le départ, sur la participation de vingt-six établissements et organismes divers à son comité intérimaire, chargé de définir sa structure, son mandat et son organisation, en plus de voir à l'obtention des fonds nécessaires à son fonctionnement. De plus, une soixantaine de personnes, actives dans le milieu de la santé, ont accepté d'emblée de siéger à ses différents comités et groupes de travail, dont les coordonnatrices et les coordonnateurs des services en français en 
place dans les établissements et organismes, les directrices et les directeurs des ressources humaines, des intervenantes et des intervenants du secteur des soins de longue durée, de même que les gens qui ont à cœur la formation de la main-d'œuvre francophone dans le domaine de la santé. U n tel intérêt pour un organisme naissant constituait une manifestation très nette du besoin pour les dirigeants et le personnel des établissements, des organismes et desinstitutions d'enseignement post-secondaire de se concerter pour répondre aux attentes de la CR SS et de la communauté.

C et enthousiasme s'est maintenu tout au long de la période de mise sur pied du R éseau. II a atteint un nouveau sommet lors de la première assemblée générale annuelle de l'organisme, le 18 février 1999, à laquelle participaient plus de 80 personnes. C inquante établissements et organismes de santé dans la région de l'Est de l'O ntario ont al ors accepté d'en devenir des membres statutaires et un premier conseil d'administration a été formé pour voir à la réalisation des objectifs du $\mathrm{R}$ éseau. Le C onseil régional de santé Champlain (CRSC) y occupe une place, conformément au protocole d'entente entre lui et le R SSFE.

\section{Les dossiers courants}

L'empressement manifesté par les établissements et lesorganismes à se joindre au R SSFE provient, en partie, de l'intérêt à l'égard des dossiers qui y sont présentement en développement. Ainsi, dès ses premiers mois d'opération, le $R$ éseau a voulu dresser un portrait des services de santé offerts sur son territoire.

II a consulté tout d'abord une cinquantaine d'études et de rapports portant sur ces services et les soins de santé dans l'Est ontarien, produits entre 1988 et 1998, et contenant des références aux services en français. L'analyse des données recueillies se poursuivra jusqu'à l'automne 1999. Le moment venu, le R éseau rendra compte de ses trouvailles et déposera un plan d'action 
concerté, valable dans le contexte actuel, pour la mise en œuvre des diverses recommandations reliées à la prestation de services de santé en français.

Le R éseau a aussi dressé un inventaire préliminaire des services et des programmes disponibles dans la région dans les domaines de la santé mentale, de la santé communautaire, de la santé publique, des soins de longue durée et des établissements hospitaliers. Plusieurs renseignements doivent encore être vérifiés, mais le R éseau espère pouvoir peindre, d'ici la fin 1999, un tableau plus juste des lacunes et des acquis du système régional de santé.

Entre-temps, les établissements hospitaliers tenus d'offrir des services en français doivent déterminer les compétences linguistiques rattachées aux postes qu'ils affecteront à ces services. Le R éseau finance et coordonne, en partenariat avec ces établissements, l'élaboration de ces profils linguistiques. En bout de ligne, on espère que cet exercice mènera à une normalisation régionale des exigences et desévaluationslinguistiques destitulai res appelés à occuper des postes désignés et à l'offre d'une meilleure qualité de services en français.

En ce qui a trait aux plans de désignation, les conseillers en développement des services en français du $R$ éseau travaillent activement avec l'H ôpital d'O ttawa, l'Institut de cardiologie de I'U niversité d'O ttawa, I'H ôpital pour enfants de l'Est de l'O ntario et le $C$ entre d'accès aux soins communautaires d' $O$ ttawa- $C$ arleton afin que ces derniers obtiennent une désignation complète.

\section{9 et au-delà}

O utre ces réalisationsà court terme, le R SSFE est aussi résolument tourné vers I'avenir. Ainsi, ses actions seront concentrées dans cinq grands secteurs au cours des prochaines années.

1. L'amélioration de l'accès aux services de santé en français dans les établissements hospitaliers et dans les organismes de soins de santé. 
L'amélioration de l'accès à une gamme complète de services de santé en français est au cœur des préoccupations du $R$ éseau. Plusieurshôpitaux de l'Est ontarien obtiendront une désignation partielle ou totale en vertu de la $L$ oi sur les services en français au cours des prochains mois (18 à 24 mois). Par ailleurs, la situation des organismes qui travaillent dans les domaines tels que la santé mentale, la toxicomanie, la santé communautaire, la santé publique et les soins de longue durée est bien différente. Plusieursorganismes se disent bilingues et ne sont pas désignés. Le R éseau aura à déterminer si leurs services sont accessibles et de qualité équivalente en français et en anglais.

2. La planification des services en français.

Le R éseau travaillera en étroite collaboration avec le C R SC au processus de planification. II définira les besoins de la clientèle francophone, identifiera les lacunes dans les services de santé en français et développera des stratégies pour répondre aux besoins.

3. La communication et le réseautage.

À ce jour, le R éseau est devenu le point d'appui du réseautage pour les intervenants et les intervenantes en santé en français. Les liens étroits qu'il a créés avec les établissements et les organismes de santé lui permettront de continuer à jouer un rôle positif à ce chapitre.

4. La formation de la main-d'œuvre francophone.

La pénurie de professionnels francophones en santé risque de nuire grandement à la capacité des établissements et organismes de santé d'assurer la prestation des services en français. Le R éseau pourra contribuer à la mise en œuvre d'un plan d'action à ce sujet, de concert avec lesinstitutions de formation post-secondaire en français et les partenaires du milieu de la santé. Cette problématique est au cœur de la réalisation des plans de désignation.

5. Le développement des services aux membres.

Le R éseau cherchera à améliorer l'accès à une gamme complète de services en français en ai dant les établissements et lesorganismes à se concerter et en leur fournissant des outils de travail et des 
services. Le R SSFE et ses membres sont bien conscients de l'ampleur de la tâche qui les attend et des attentes de la communauté francophone. Pourtant, la volonté manifeste de tous et chacun de relever les défis énumérés dans cet article est déjà un gage de succès.

\section{Note}

1. Pour communiquer avec le R éseau, vous pouvez utiliser un des moyens suivants: par poste, à R éseau des services de santé en français de l'Est de l'O ntario, 1173, chemin Cyrville, bureau 300, G loucester, 0 nt. K1J 7S6; par courrier électronique à reseau@ rsffe.on.ca; par téléphone au (613) 747-7431 et par télécopieur au (613) 747-2907 